\title{
Sterile keratitis post collagen cross-linking (CXL) therapy for treatment of keratoconus
}

\author{
Ankur Mehta ${ }^{1}$, Richard Barry' ${ }^{1}$, Simon Liew ${ }^{2}$, Nathanial Knox-Cartwright ${ }^{1}$ \\ 'Sydney Eye Hospital, Sydney, Australia; ${ }^{2}$ Royal Prince Alfred Hospital, Sydney, \\ Australia
}

\begin{abstract}
Corneal collagen cross-linking therapy is a relatively new treatment for progressing corneal ectasias such as keratoconus. Here the authors discuss the case of a 22-year-old male who underwent collagen cross-linking for treatment of progressive keratoconus, who then developed a serious post operative complication of a sterile keratitis.
\end{abstract}

Key words: Collagen cross-linking, keratoconus, sterile keratitis

\section{Introduction}

Corneal collagen cross linking therapy is a relatively new treatment for progressing corneal ectasias such as keratoconus. The concept is to strengthen the cornea by inducing stronger bonds between collagen fibres in the corneal stroma. ${ }^{1}$ As initially described, UV A radiation is applied for 30 minutes to a de-epithelialised cornea that has been saturated with riboflavin. In the original trials no adverse effects were reported, but the authors were keen to note that as the technology and its application were new, practioners should only use this treatment for documented progressive disease only, until further experience and data were collected. ${ }^{1}$

Since its introduction, further studies have attempted to assess the safety and efficacy of the treatment. ${ }^{2,3}$ It is considered a safe technique for the operator, ${ }^{4}$ however, there are now case reports suggesting some complications associated with this treatment. $^{5-7}$ These complications may be associated with certain steps in the procedure, de epithelialisation or irradiation of the cornea, or a combination of steps.

Here we will discuss the case of a patient who underwent CXL for treatment of progressive keratoconus, and presented two days post treatment with a significant complication from the procedure.

\section{Case report}

A 22-year-old male of Fijian-Indian background presented to our clinic less than 48 hours post CXL treatment for keratoconus in the right eye (RE). He reported bilateral keratoconus, with previous hydrops in the left eye (LE). He reported no previous surgery to the RE or treatment to the RE. There was documented progression of his keratoconus in the right eye and he underwent CXL treatment. The technique used followed that published by Wollensak et al. ${ }^{1}$ After the treatment, a bandage contact lens was applied and he was commenced on ketorolac trometamol (Acular $5 \mathrm{mg} / 5 \mathrm{ml}$ ) TDS and Tobramycin 0.3\% (Tobrex) TDS.

Correspondence: Dr. Ankur Mehta, Sydney Eye Hospital, Sydney, NSW 2000, Australia. E-mail:anx01@hotmail.com 
On presentation, his visions were RE hand movements only (HM) and LE $<6 / 60$. On examination, the contact lens was not found. His RE showed severe ciliary injection, slit lamp microcopy revealed a large central epithelial defect, multiple small to medium sized corneal infiltrates, posterior synechiae and an endothelial plaque. The anterior chamber showed $3+$ cells, consistent with an acute keratouveitis. The LE showed a central scar, with central stromal thinning (Fig. 1).

Initial management consisted of corneal scrape and swab to attempt to identify causative pathogen (MC\&S including fungal culture, PCR for HSZ/VZV) and commencement of intensive presumptive treatment. This consisted of cephazolin $5 \%$ eye drops, gentamicin $0.9 \%$ eye and natamycin $5 \%$ eye drops hourly in the RE. He was also commenced on oral voriconazole ( $400 \mathrm{mg} \mathrm{BD}$ for $24 \mathrm{hrs}$ as induction, then reduced to $200 \mathrm{mg} \mathrm{BD}$ for maintenance) Ciprofloxacin (750 mg BD orally) and atropine $1 \%$ eye drops BD.

Microscopy showed a gram-negative organism, indicating a bacterial keratitis as the main differential. In light of this, his antibiotic regimen was changed from cepahazolin $5 \%$ eye drops to ceftazidime $5 \%$ eye drops for better gram-negative cover.

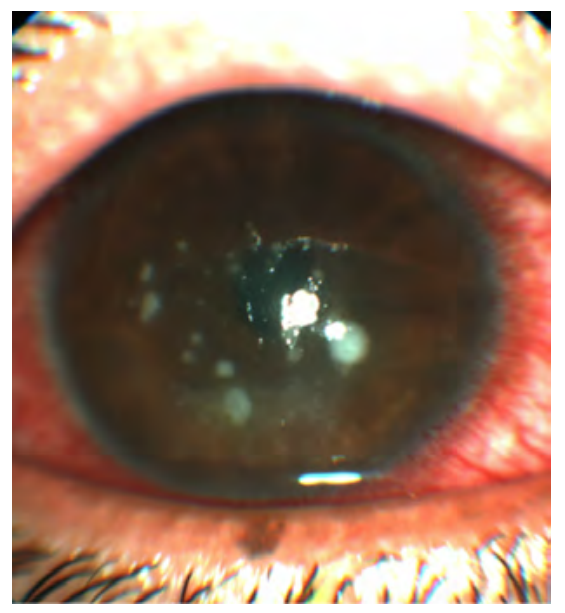

Fig. 1. Photograph of RE on presentation. After seven days of treatment there was no clinical improvement. Final microbiology analysis suggested that the initial gram-negative source was likely due to sampling contamination; final culture studies and PCR testing were negative. Examination of the RE revealed persistent anterior stromal infiltrates and white cell recruitment (WCR) centrally, and no change in the epithelial defect.

To identify the causative pathogen, confocal microscopy and corneal biopsy were performed post cessation of antibiotics for $24 \mathrm{hrs}$. Figure 2 shows the confocal microscopy image, this was reported as hyper-intense cyst-like lesions in deep stroma. This was consistent with Acanthamoeba keratitis. In light of this, the patient was commenced on PHMB (Polyhexamethylene biguanide) and brolene eye drops hourly. 


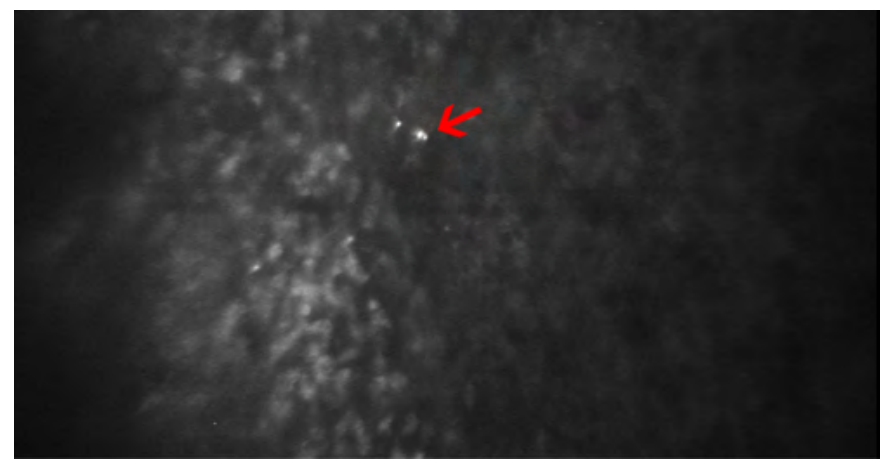

Fig. 2. Confocal microscopy image showing cyst.

Over the following days, the patient reported less pain in the RE. On examination the ciliary injection began to improve. On day 13, the appearance of the infiltrates improved, but the epithelial defect remained unchanged (Fig. 3). Corneal biopsy results were returned and were negative for Acanthamoeba. In light of the partial response to PHMB treatment, it was decided to continue this in the short term.

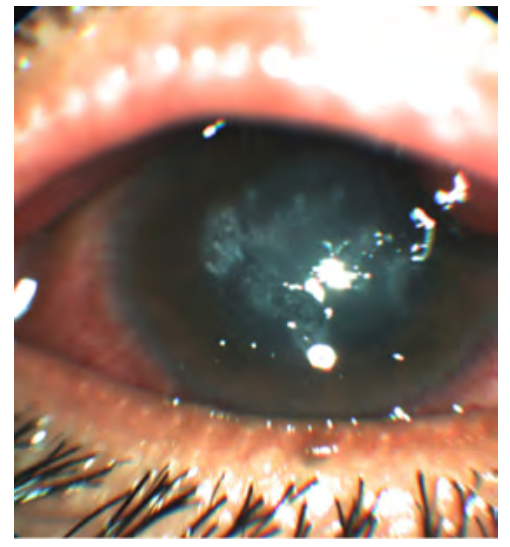

Fig. 3. Photograph of RE cornea at day 13.

The patient was discharged with outpatient follow-up, but at three days post discharge he reported worsening pain. He was referred to a specialized eye hospital. Examination showed that the epithelial defect was worse, with persistent central stromal infiltrates. All his drops were ceased for 24 hours and further corneal scrapings were obtained and sent for microbiological assessment.

Repeat corneal cultures and PCR were performed; results were negative for micro-organism, fungi and HSV PCR. He was then commenced on a topical steroid, and was monitored. He showed improved corneal appearance. Unfortunately, the patient self-discharged. He re-attended for follow-up approximately two weeks post self-discharge, on examination he had re-epithelialized the cornea, but best corrected vision was unchanged at hand movements only in the RE. 


\section{Discussion}

The initial presentation, in regards to the onset post procedure, presents limited differentials. There have been reports of bacterial keratitis and infiltrates post CXL. ${ }^{8-10}$ In these reports, causative organisms have penetrated the to the stroma of the cornea due to the absence of the protective layers of the cornea which are removed to allow absorption of the riboflavin or through intra or early post procedural contamination. These cases present with similar infiltrates, and in a similar time frame, but in the absence of a clear causative pathogen (initial gram negative was thought to be due to sample contamination rather than the causative organism of the keratitis) and the slow response to treatment targeting presumptive organisms, a hypersensitivity reaction to the either the riboflavin or the UVA radiation source was suspected to have caused the keratitis. Sterile keratitis is a known complication of $\mathrm{CXL}^{11,12}$ but sterile infiltrates are associated with contact lens wear, autoimmune disease, immune mediated local reaction, non steroidal anti inflammatory drug use and corneal surgery. This patient had some of these risk factors, namely the commencement of NSAID drops post procedure (Acular) and placement of bandage contact lens. ${ }^{11}$ In this case we cannot rule out a causative organism that was not detected by standard culture media, but sensitive to the prescribed treatment.

The confocal microscopy complicated, rather than contributed to diagnostic process. There have been reported cases of $A K$ post $\mathrm{CXL}^{7}$ but definitive exposure to tap water was noted in the presenting history. The onset of our patient's symptoms was not consistent with AK. AK is usually seen as a progressive, slow onset with atypical features of bacterial or fungal keratitis. ${ }^{13}$ The appearance of cysts on the confocal microscopy, in the absence of positive tissue diagnosis presented a diagnostic quandary. The diagnosis of AK by PCR has been shown to have a sensitivity of $80 \%$ and a specificity of $100 \%$ in one study, ${ }^{14}$ and in another study 24 out of 31 cases $(77.4 \%)$ of AK diagnosed by confocal microscopy were confirmed by PCR. ${ }^{15}$ Tissue diagnosis with PCR remains the gold standard for diagnosis of AK.

The apparent response to treatment after the introduction of PHMB is difficult to assess given the entirety of the treatment regimen at that stage. The response was marginal, and may not indicate treatment of AK.

Arora et al. ${ }^{12}$ describe a similar progression in their case study of a child post CXL for progressive keratoconus. In their case, there was no confounding confocal microscopy finding and they instigated a regimen of topical steroid early in the presentation, within 48-72 hours post CXL procedure, with good results. In another case, corneal infiltrates were seen on day 5 post $\mathrm{CXL}^{16}$ and topical steroids were commenced to good effect. Sterile corneal infiltrates have been described in several clinical settings and probably occur as a result of enhanced cell mediated immunity to staphylococcal antigens deposited in high concentrations in areas of static tear pooling. ${ }^{12,16}$

CXL is an increasingly popular treatment for progressive keratoconus. It is growing in popularity, and the body of literature surrounding CXL is growing. Our experience highlights a potential risk of sterile keratitis after CXL treatment. 


\section{References}

1. Wollensak G, Spoerl E, Seiler T. Riboflavin/ultraviolet-A-induced collagen cross linking for the treatment of keratoconus. Am J Ophthalmol 2003;135:620-627.

2. Goldich YMD, Marcovich ALMD, Barkana YMD, Mandel YMD, Hirsh AMD, Morad YMD, et al. Clinical and Corneal Biomechanical Changes After Collagen Cross-Linking With Riboflavin and UV Irradiation in Patients With Progressive Keratoconus: Results After 2 Years of Follow-up. Cornea 2012;31(6):609-614.

3. Goldich YMD, Marcovich ALMD, Barkana YMD, Avni IMD, Zadok DMD. Safety of Corneal Collagen Cross-linking With UV-A and Riboflavin in Progressive Keratoconus. Cornea 2010;29(4):409-411.

4. Shetty RM, Shetty RDNBF, Mahendradas PDODNB, Shetty BKMS. Are the Surgeons Safe During UV-A Radiation Exposure in Collagen Cross-Linking Procedure? Cornea 2012;31(2):167-171.

5. Garcia-Delpech S, Diaz-Llopis M, Udaondo P, Salom D. Fusarium keratitis 3 weeks after healed corneal cross-linking. J Refract S 2010;26(12):994-995.

6. Bagga B, Pahuja S, Murthy S, Sangwan VS. Endothelial failure after collagen cross-linking with riboflavin and UV-A: case report with literature review. Cornea 2012;31(10):1197-1200.

7. Rama P, Di Matteo F, Matuska S, Paganoni G, Spinelli A. Acanthamoeba keratitis with perforation after corneal crosslinking and bandage contact lens use. J Cataract Refract Surg 2009;35(4):788-791.

8. Pollhammer M, Cursiefen C. Bacterial keratitis early after corneal crosslinking with riboflavin and ultraviolet-A. J Cataract Refract Surg 2009;35(3):588-589.

9. Sharma N, Maharana P, Singh G, Titiyal JS. Pseudomonas keratitis after collagen crosslinking for keratoconus: case report and review of literature. J Cataract Refract Surg 2010;36(3):517-520.

10. Perez-Santonja JJ, Artola A, Javaloy J, Alio JL, Abad JL. Microbial keratitis after corneal collagen crosslinking. J Cataract Refract Surg 2009;35(6):1138-1140.

11. Rodriguez-Ausin P, Gutierrez-Ortega R, Arance-Gil A, Romero-Jimenez M, Fuentes-Paez G. Keratopathy after cross-linking for keratoconus. Cornea 2011;30(9):1051-1053.

12. Arora R, Jain P, Gupta D, Goyal JL. Sterile keratitis after corneal collagen crosslinking in a child. Contact Lens \& Anterior Eye 2012;35(5):233-235.

13. Dart JKG, Saw VPJ, Kilvington S. Acanthamoeba keratitis: diagnosis and treatment update 2009. Am J Ophthalmol 2009;148(4):487-499.e2.

14. Lehmann OJ, Green SM, N M. polymerase chain reaction analysis of corneal epitheilial and tear samples in the diagnosis of acanthamoeba keratitis. Invest Ophthalmol Vis Sci 1998(39):1261-1265.

15. Mathers WD, Nelson SE, Lane JL, Wilson ME, Allen RC, R F. Confirmation of confocal microscopy diagnosis of acanthamoeba keratitis using polymerase chain reaction analysis. Arch Ophthalmol 2000(118):178-183.

16. Angunawela RI, Arnalich-Montiel F, Allan BDS. Peripheral sterile corneal infiltrates and melting after collagen crosslinking for keratoconus. J Cataract Refract Surg 2008;35(3):606-607. 